\title{
¿Qué clase de creencias es posible atribuirle al escéptico pirrónico?
}

\author{
Luis Ángel García Muñoz \\ Facultad de Filosofía y Letras \\ Universidad Nacional Autónoma de México \\ garcialuisangel87@gmail.com
}

\section{Introducción}

Sexto Empírico define su escepticismo como «la capacidad de establecer antítesis en los fenómenos y en las consideraciones teóricas, según cualquiera de los tropos; gracias a la cual nos encaminamos - en virtud de la equivalencia entre las cosas y proposiciones contrapuestas - primero hacia la suspensión del juicio y después hacia la ataraxia» (Sexto, Libro I, cap. IV: 8). La suspensión del juicio refiere a lo que llamaremos epoché, y la ataraxia al «bienestar y serenidad del espíritu» (Sexto, Libro I, cap. IV: 10). De lo anterior obtenemos los siguientes supuestos:

(1) La ataraxia es el objetivo último del escepticismo en los términos de Sexto.

(2) La epoché es el paso anterior a la ataraxia.

(3) Los tropos son el medio para llegar a la epoché. 
Sobre (b), Hankinson aclara que su consecuencia es incierta. Existe la posibilidad de que la suspensión del juicio no conduzca a la ataraxia (Hankinson, 1995: 141), por lo que el método escéptico no funciona para todos de la misma manera.

Sobre (c) tenemos diez tropos que nos ayudan a llegar a (b) estableciendo la equivalencia entre las cosas y las proposiciones contrapuestas; y tenemos cinco modos aún más sofisticados con la misma finalidad que denominamos «de Agrippa». Hankinson formaliza los primeros de la siguiente manera:

\section{[A]}

(1) $x$ parece $F$ en relación a $a$.

(2) $x$ parece $F^{\prime}$ en relación a $b$.

(3) Al menos una de las apariencias (1) o (2) puede ser verdadera.

(4) Ningún criterio nos ayuda a decidir entre (1) y (2).

(5) No podemos establecer un juicio acerca de lo que $x$ es en su naturaleza real (ver Hankinson, 1995: 139).

[A (4)] parece que no adquiere la fuerza suficiente para el argumento desde que no está justificada y se adopta como un supuesto. Sin embargo, su validez se puede complementar con los argumentos en contra del criterio que ofrece Sexto (ver Sexto, Libro II, cap. III-VII). El argumento inicial puede ser expuesto de la siguiente forma:

\section{[B]}

(1) Existe un criterio.

(2) No existe un criterio.

(3) Si la disputa entre (1) y (2) no se puede resolver, se suspende el juicio sobre la verdad de (1) o de (2). 
(4) Si la disputa entre (1) y (2) se puede resolver, entonces necesitamos un criterio para resolver la disputa.

(5) No contamos con un criterio para resolver la disputa, pues el criterio es aquello que se disputa.

(6) Si se llegara a establecer un criterio hipotético primario que determine otros criterios, éste necesitará a su vez una garantía, y la garantía necesitará a su vez otra garantía, y así hasta el infinito.

El paso [B (5)] es un círculo vicioso desde que aquello que quiere demostrarse necesita una garantía derivada de lo que se está estudiando. El paso [B (6)] cae en un regreso al infinito. De la validez de [B] depende que [A (4)] esté justificado.

El círculo vicioso y el regreso al infinito son dos de los cinco modos de Agrippa que se exponen brevemente de la siguiente manera:

a) De la disputa:

1) Si hay una divergencia de opiniones respecto a un tema,

2) y no podemos elegir ni rechazar ninguna opción,

3) suspendemos el juicio.

b) De la relatividad:

1) Los objetos aparecen siempre en relación con el que juzga, o en relación con el medio,

2) por lo que el objeto no se presenta en sí.

3) no conocemos su naturaleza y anulamos el juicio sobre la misma.

c) Círculo vicioso:

1) $S$ afirma que $p$

2) $p$ está basado en $q$

3) q está basado en $p$

4) $p$ está basado en $q$ 
d) Regreso al infinito:

1) La cuestión 1 está garantizada por $A$

2) La garantía $A$ está garantizada por $B$

3) La garantía $B$ está garantizada por $C$. Así hasta el infinito.

4) No sabemos dónde inicia la argumentación,

5) y suspendemos el juicio.

e) Por hipótesis:

1) Se establece una hipótesis que pretende poner fin al regreso al infinito,

2) y la hipótesis carece de una garantía evidente $X$, por lo que no está demostrada,

3) entonces, la hipótesis se torna inválida por falta de justificación suficiente y se anula el juicio.

Los diez tropos, más los cinco expuestos arriba, tienen como objetivo suspender el juicio; esto es: no rechazar ni afirmar cosa alguna. Sexto dice refiriéndose a los primeros diez tropos: «el fundamento de la construcción escéptica es ante todo que a cada proposición se le opone otra proposición de igual validez. A partir de esto, en efecto, esperamos llegar a no dogmatizar» (Sexto, Libro I, cap. VI: 12). Barnes, quien estudia el sentido de histórico de dogmata, nota que el término puede referir a i) creencias importantes o ii) creencias de naturaleza práctica (Hankinson, 1995: 248). De aquí que algunos, como Hankinson, interpreten que el escéptico tiene el objetivo de no tener creencia alguna. Mi postura es que esta visión es errónea en cuanto que el escéptico (como Sexto lo entiende), pese a los tropos, tiene creencias prácticas.

En lo que sigue, esbozaré algunos argumentos con distintas nociones de creencia y veremos cuál adoptaría el escéptico pirrónico. Lo anterior para dilucidar bajo qué criterios 
se le puede adjudicar creencias al escéptico pirrónico y bajo cuáles no.

Frede: creencias epistémicas y no epistémicas

Frede acepta que el escéptico pirrónico no tiene creencias en el sentido en que no hay una doctrina sistemática que todo escéptico pirrónico acepte (Frede, 1987: 179). Sin embargo, les adjudica cierta clase de creencias a los escépticos (que siguen a Sexto y no a Pirrón). En lo que sigue, Frede mostrará que i) Sexto no separa la vida de las creencias, como se cree que hizo Pirrón, y que ii) esas creencias son compatibles con el escepticismo desde que carecen de un sentido epistemológico similar al de los dogmáticos.

Frede inicia con la intuición de que es generalmente asumido que la vida ordinaria no es posible sin creencias (ver 1987: 180). Si el escéptico a través de los tropos trata de suspender las creencias, entonces tenemos dos opciones:

1) El escéptico, al no tener creencias, no puede vivir por lo menos en condiciones normales; por lo que tendría una vida sin creencias (ver 1987: 181).

2) El escéptico, a pesar de que ha suspendido el juicio en todas las materias, tendrá creencias suficientes que le permitan tener una vida bajo condiciones normales (ver 1987: 181).

La primera opción, dice Frede, caracteriza los relatos acerca de Pirrón; la segunda resume la actitud de Sexto. Antigonus de Carystus, biógrafo y contemporáneo de Pirrón, lo describe como alguien que vivía sin creencias desde que no evitaba ningún riesgo ni confiaba en los sentidos, a tal grado de que 
sus seguidores siempre lo rescataban de que se causara algún daño mientras caminaba por la calle (1987: 181). Debido a que Pirrón es la figura emblemática del escepticismo pirrónico, se ha generalizado su influencia a tal grado de afirmar que todo escéptico pirrónico vive sin creencias. Sin embargo, Sexto es un caso distinto, e incluso, es pertinente ser precavido cuando se le denomina pirrónico. La vida escéptica, enfatiza Sexto, debe ser convencional (1987: 182), lo que implica tener costumbres que lo distanciarían del modo de vida de Pirrón. De esto, y del supuesto de que las acciones presuponen creencias (ver 1987: 184), se sigue que Sexto tenía una vida con creencias. Cuando Sexto, explica que el escéptico no dogmatiza, distingue dos sentidos de dogmata:

1) «Dogma es aprobar algo en términos más o menos generales».

2) «Dogma es la aceptación en ciertas cuestiones, después de analizadas científicamente, de cosas no manifiestas» (Sexto, Libro I, cap. VII, 2002: 13).

Añade que el pirrónico no asiente ninguna de las cosas no manifiestas; y que a su vez no dirá cosas como: «creo que no siento frío» o «no siento frío» (Sexto, Libro I, cap. VII: 13); lo que nos indica que el escéptico no dogmatiza en el segundo sentido, pero sí en el primero.

Frede (1987: 186 y 187) se pregunta qué clase de creencias son aquellas del primer sentido, si suponemos que el escéptico suspende el juicio de todas ellas; y responde de la siguiente manera:

1) Hacer un juicio acerca de aquello que las cosas «parecen» es distinto de hacer un juicio sobre aquello que las cosas «son». 
2) El escéptico pirrónico, por lo menos Sexto, puede hacer juicios en el sentido (de lo que las cosas «parecen»), pero no los hace en el sentido (de lo que las cosas «son»).

3) «Parecer»y «aparecer» carecen de un sentido epistemológico, según Frede, desde que «parece que $p$ » no dice «creo que $p$ es el caso»; sino «parece como si $p$ fuera el caso».

4) Por «sentido epistemológico» Frede entiende aquellos casos donde la representación mental es directamente accesible.

De lo anterior, Sexto tiene claramente una restricción hacia los juicios en el sentido de lo que las cosas «parecen», ya que para el escepticismo de Sexto es primordial distinguir el estatuto de una apariencia y de la realidad; donde la segunda no nos es manifiesta. Sexto sólo hace juicios acerca de lo que le es evidente; es decir, acerca de lo que le parece en relación sus sentidos, con persona, situación, locación y costumbres.

Para Frede, es evidente que a Sexto se le pueden atribuir creencias siempre y cuando éstas sean en un sentido no-epistemológico que obedezcan a los juicios acerca de las apariencias de las cosas. Caso contrario al de Hankinson, como se explicará a continuación.

Hankinson: creer $v s$ realizar actos de aceptación

Hankinson responde a si los escépticos pirrónicos tienen creencias de una manera similar, mas no idéntica, a la de Frede. Concuerdan en la diferencia entre «creo que $p$ es el caso» $\mathrm{y}$ «parece como si $p$ fuera el caso»; sin embargo, Hankinson no le atribuye creencias al escéptico, sino sólo actos de aceptación o asentimiento. Hankinson (1995: 249 y 250) lo argumenta de la siguiente manera: 
1) El supuesto de Frede referente a que las acciones presuponen creencias es falso, según Hankinson, desde que: podemos actuar como si creyéramos en algo, y permanecer agnósticos a la vez en casos donde aceptamos conductivamente y resguardamos la paz mental. Ejemplo: adorar a Dios por costumbre o cultura, y permanecer agnóstico al tema de su existencia.

2) El anterior ejemplo no muestra que «apariencia» es distinto de «creencia», sino que distingue una regla pragmática (adorar a Dios por costumbre), de una verdad metafísica (si Dios existe o no).

3) La regla pragmática es un acto de aceptación que no está comprometido con la verdad (en el sentido metafísico). Pero una creencia siempre es un juicio de algo que es verdadero; por lo que un acto de aceptación es distinto a una creencia. De aquí, Hankinson propone la siguiente división:

Creencia: estado mental sobre algo que es verdadero.

Acto de asentir: acto mental en el que nos inclinamos al contenido de una impresión que es proposicional en forma, y que está acompañada de un impulso.

La definición (i) muestra una clara crítica a Frede desde que para Hankinson una creencia siempre es epistémica, por lo que no habría tal cosa como una «creencia en un sentido no epistemológico»; la disputa se centra sobre definiciones divergentes de «creencia». Hankinson (1995: 251) toma la definición (ii) de los estoicos y agrega el uso que le daban al término:

1) Los estoicos distinguen animales de humanos en cuanto a la interposición de asentir a las impresiones y las acciones. Mientras los segundos pueden evitar actuar, los primeros no. 
2) Un ejemplo de un impulso sería «comer la manzana»; un ejemplo de una acción, que se caracteriza por ser normativa es: «comer la manzana es bueno para mí». El estoico propone que los humanos pueden evitar actuar a partir de las anteriores proposiciones desde que puede considerar lo que es genuinamente un deseo, $\mathrm{y}$ lo que no es. Los animales no pueden evitar actuar, desde que no consideran lo que es genuinamente un deseo.

Sexto anula la separación entre animales y humanos. «Asentir» cobra un uso distinto para él:

1) El escéptico asiente sobre las afecciones de las impresiones.

2) Actúa de acuerdo a las impresiones.

3) Si asentir implica comprometerse con que lo asentido es verdadero o falso, el escéptico no asentirá (ver 1995; 252).

4) Sin embargo, por (1) y (2), el escéptico asiente (ya que actúa), pero no está comprometido con la verdad o falsedad de aquello que asiente.

5) Hankinson afirma que «asentir» es un estado mental que difiere de «creer» y de sus implicaciones epistemológicas. Esta diferencia es lo que le permitiría a Sexto no comprometerse con la posibilidad de dogmatizar acerca de sus impresiones.

Hankinson (1995: 253-255), para fundamentar (5), describe la vida mental del escéptico donde, en última instancia, trata de mostrar que es posible llevar una vida sin «creencias» en el sentido que él propone. Para ello, nota que una vida sin creencias debe suponer los siguientes puntos:

1) El escéptico tiene acceso privilegiado a sus propias afecciones, incluso si es o no apropiado describirlas en términos de falsedad y verdad. 
2) El criterio del modo de vida del escéptico son las apariencias; esto es, las impresiones que se asienten.

3) El contenido de los actos de asentir es pragmático, no normativo.

4) El contenido normativo está comprometido con una «creencia» desde que está comprometido con descripciones acerca de lo verdadero. El contenido pragmático, según Hankinson, está comprometido con lo que denomina «asentir».

5) El escéptico basa su comportamiento en impresiones; sin embargo, hay dos clases de impresiones:

i) Impresiones de primer orden comprometidas con lo que siente (tener frío, percibir lo dulce de la miel).

ii) Impresiones de segundo orden comprometidas con las apariencias correspondientes a la moral (si fumo un cigarrillo y noto que es ofensivo para mi acompañante, lo apago).

El estado mental del escéptico difiere del estado mental del dogmático en que el primero no tiene creencias, sino que sólo realiza actos de asentimiento basados en impresiones sin establecer su verdad o falsedad; mientras que el segundo tiene creencias en el sentido en que realiza juicios acerca de lo que es verdadero.

El escéptico, en cuanto a la comparación con los estoicos, asiente sobre impresiones igual que un animal podría asentir sobre ellos. Queda pendiente que Hankinson dé respuesta a si los animales tendrían, a su vez, impresiones de primer y segundo orden.

Hasta ahora vemos que mientras Frede le adjudica creencias a Sexto, Hankinson sólo le atribuye actos de asentimiento. La disputa, como se menciona, parte de una noción distinta sobre «creencia». 


\section{Cohen: aceptación vs creencia}

En un tono similar al de Hankinson, Cohen trata de mostrar que existen diferencias entre dos estados mentales: «aceptar» $\mathrm{y}$ «creer». Debido a que el conocimiento proporcionado por la folk psychology - que describe la mente y sus estados a través del sentido común y de los distintos usos de términos como «creencia»y «deseo»— no resuelve, para Cohen, algunos problemas de origen epistemológico y lingüístico, la diferencia entre el estado mental de «creencia» y el estado de «aceptación» se vuelve no sólo interesante, sino necesaria (ver Cohen, 1992: 3). Cohen (1989: 368-369) define a cada estado con las siguientes características:

a) Sobre el acto de «aceptar»:

1) Es un acto mental, o bien una política sobre acción mental; esto es: implica una acción en el campo conductual, y no sólo un acto discursivo.

2) «Aceptar que $p$ » implica un compromiso voluntario hacia $p$; esto es: implica un compromiso voluntario para actuar de manera conductual en relación a $p$.

3) No tiene un compromiso meramente epistemológico desde que no está comprometido con la verdad o falsedad de $p$.

b) Sobre el acto de «creer»:

1) Es una disposición a sentir que $p$ es verdadera o falsa.

2) Por «sentir» podemos entender estar convencido de que $p$ es verdadera; o estar incluso sorprendido de que $p$ resulte falsa'.

\footnotetext{
${ }^{1}$ La definición de «creer» de Cohen puede ser explicada, pero no reducida a la definición de Davidson de «creencia» desde que la primera incluye un «sentimiento» y la segunda una «actitud». Si atendemos la definición de creencia como una «actitud proposicional» de Davidson, explicamos que el sujeto [S] tiene una actitud [A] sobre una proposición [P]; esto es: Carlos espera que llueva mañana. De la misma manera, para Cohen se establece un
} 
3) «Creer que $p »$ no es un acto voluntario desde que no podemos voluntariamente no creer algo de lo que nos han mostrado evidencia irrefutable.

Cohen propone el siguiente caso para distinguir ambos estados mentales: «Por propósitos profesionales, un abogado puede aceptar que su cliente no es culpable, incluso si no lo cree» (1989: 369). El sujeto «acepta» en cuanto lo hace voluntariamente, y está comprometido a actuar en relación con dicha proposición. No «cree» la proposición en cuanto está comprometido de manera epistémica sobre la verdad o falsedad de la proposición. Su creencia no es voluntaria en cuanto parece que hay evidencia lógica o material que lo obligan a tener ese estado mental. Como el anterior ejemplo muestra, se pueden tener ambos estados mentales a la vez; es decir, los estados mentales no están en categorías distintas desde que ambos pueden interactuar en una misma situación. El anterior ejemplo no se puede explicar por las definiciones del sentido común sobre la mente — folk psychology— según Cohen.

Al igual que Hankinson, Cohen podría describir la vida sin creencias del escéptico con la anterior distinción de estados mentales. Mientras el escéptico «acepta» proposiciones y actúa en relación con ellas, puede no estar comprometido con la verdad o falsedad de las mismas. 


\section{Folk psychology}

Lo primero que hay que notar sobre la folk psychology es que no está comprometida con ninguna definición teórica sistemática de actos mentales como «creer», «asentir», «aceptar» o «desear» como fue el caso de Frede, Hankinson y Cohen. La folk psychology tampoco tiene una definición sistemática de sí misma. De hecho, su significado se puede establecer por distintos usos en la literatura filosófica (Ravenscroft, 2010):

1) Se usa para referir cierto conjunto de capacidades cognitivas que incluyen las capacidades de predecir y explicar el comportamiento.

2) Se puede usar para referir la teoría del comportamiento representado en el cerebro.

3) Y por último, se usa para referir una teoría psicológica constituida por los distintos usos obvios de términos como «creencia»o «deseo», que comúnmente son aceptados por la mayoría.

Nosotros nos referiremos al tercer uso, ya que presenta una gran gama de posibles descripciones sobre el término «creer». Las descripciones, al depender de los diversos usos, no se comprometen con distinciones elegantes entre «creer» y «aceptar», o entre «creer epistemológicamente» $\mathrm{y}$ «creer no-epistemológicamente». Lo anterior permite analizar los distintos usos que implican los términos, y de ahí establecer significados contextuales; procedimiento posiblemente compatible con los análisis lingüísticos del segundo Wittgenstein.

Guillermo Hurtado, en su artículo «Dudas y sospechas», aclara que su intención no es manejar una definición de «creencia», sino que, al analizar distintos usos del término, establece que podemos distinguir grados de creencia desde 
que hay usos donde nuestra creencia de $p$ parece algo indubitable [Hurtado le llama «certeza» (ver 2009: 65)], y hay otros usos donde afirmamos $p$ pero con cierta reserva. Para el primer caso obedeceríamos a la forma: «creo que $p$ es verdadera»; y en el segundo obedeceríamos: «creo que $p$, pero creo que $p$ puede resultar falsa». En ambos casos creemos en algo, pero el término cobra distintos significados e implicaciones. Mientras en el primero estamos comprometidos con $p$, con defender su verdad o falsedad, en el segundo caso creemos $p$ sin certeza. La propuesta de Hurtado necesita contraponer una visión clásica de «creencia» con una descripción de los distintos usos del término «creencia». Desde que Hurtado opta por la segunda y no se compromete con alguna visión rígida del término, podríamos decir que su visión es compatible con el tercer uso de la folk psychology. El discurso de Hurtado procede de la siguiente manera:

1) La epistemología clásica describe de la siguiente manera los términos i) «creencia» y ii) «duda»:

i) «creo que $p$ es verdadera» $\mathrm{o}$ «tengo la certeza de que $p$ es verdadera».

ii) «dudo que $p$ » «suspendo el juicio sobre si $p$ es verdadera o falsa».

2) Para la epistemología clásica, la «creencia» y la «duda» son contrarias; por lo que no aceptaría casos bajo la forma: «creo que $p$, aunque dudo que $p »$ (Hurtado, 2009: 66).

3) Hurtado nota que hay casos donde no es contradictorio sostener que se cree que $\mathrm{p}$, pero se duda que $\mathrm{p}$ :

i) «Creo en Dios todopoderoso, pero tengo mis dudas sobre su existencia».

ii) «Creo que cerré la puerta con llave, pero no estoy seguro». 
En 3) los casos sugieren que la $d u d a$ depende de un grado de justificación sobre $p$ (Oakey, 1988). Incluso se ha propuesto un sistema gradual de justificación basado en la probabilidad (Lina \& Hájek, 2007). Podemos creer $p$, sin tener una justificación suficiente de $p$, por lo que aún podemos tener dudas sobre $p$. Hurtado dice: «Un teólogo puede tener muchas preguntas sin respuesta sobre Dios y, sin embargo, su creencia en él puede ser tan sólida como una roca» (2009: 68). De lo anterior podemos rescatar que:

1) Hay casos donde es posible creer $p$ sin justificación necesaria sobre $p$.

2) Hay casos donde creemos $p$, aunque tengamos algunas dudas sobre $p$.

3) Hay casos donde dudar que $p$ implica creer que $p$ : Dudamos sobre el aspecto 1 de $p$, y aún así creemos que $p$.

Los anteriores supuestos sólo tienen validez si no jugamos bajo los términos de la epistemología clásica que describe, define y restringe los casos donde se «cree» y se «duda», colocándolos como estados mentales contradictorios. De hecho, aquellos que cuestionan la actitud escéptica de una vida sin creencias presuponen que la «duda» o la «suspensión del juico» es contraria a «creer», por lo que eliminan las creencias de la vida escéptica. Sin embargo, si no partimos de una definición estática de «creer» y optamos por los distintos significados que adquiere el término dependiendo del uso, veremos que es posible decir que el escéptico «cree en $p$, pero suspende el juicio (o tiene dudas) sobre $p »$; ejemplificando: el escéptico cree en las apariencias del agua fría $(p)$; pero suspende el juicio sobre un aspecto de $p$ no justificado 
o justificado débilmente, esto es: que de hecho el agua sea esencialmente fría.

La folk psychology no tendría problemas en atribuirle creencias al escéptico siempre y cuando se restrinjan los usos donde «creer» implique apostar indubitablemente por la verdad o falsedad de $p$.

\section{¿Qué noción de «creencia» es compatible con la actitud del escéptico?}

Se han expuesto distintas nociones de «creencia», de las cuales se presenta la siguiente tabla. Lo que resta es ver cuál de ellas es compatible con la actitud escéptica, particularmente con la de Sexto Empírico.

\begin{tabular}{|l|c|c|c|}
\cline { 2 - 4 } \multicolumn{1}{c|}{} & $\begin{array}{c}\text { Estados que tie- } \\
\text { nen un compromi- } \\
\text { so con la verdad o } \\
\text { la falsedad de } p\end{array}$ & $\begin{array}{c}\text { Estados que no } \\
\text { tienen un compromi- } \\
\text { so con la verdad o la } \\
\text { falsedad de } p\end{array}$ & $\begin{array}{c}\text { Ninguna } \\
\text { de las } \\
\text { anteriores }\end{array}$ \\
\hline Frede & $\begin{array}{c}\text { «Creer epistemo- } \\
\text { lógicamente» }\end{array}$ & $\begin{array}{c}\text { «Creer no-epistemoló- } \\
\text { gicamente» }\end{array}$ & ----- \\
\hline Hankinson & «Creer» & «Aceptar» «Asentir» & ---- \\
\hline Cohen & «Creer» & «Aceptar» & ---- \\
\hline $\begin{array}{l}\text { Folk } \\
\text { Psychology }\end{array}$ & ----- & ---- & «Creer» \\
\hline
\end{tabular}

Si la definición de Frede fuera compatible con la actitud escéptica de Sexto:

1) Sexto sólo tendría creencias en un sentido no epistemológico, es decir, tendría creencias en el sentido que las representaciones 
mentales no son directamente accesibles; esto es: tendría creencias sólo de apariencias, no de realidades.

2) Sexto estaría comprometido con una definición de aquello que es «epistemológico»y aquello que no es «epistemológico».

3) Sexto podría dar razón del siguiente pasaje (al que nos referiremos en adelante como « $\alpha »)$ donde afirma que los escépticos tienen creencias (dogmata) sólo en un sentido:

$\alpha$ : Que el escéptico no dogmatiza, y no lo decimos en el sentido de dogma en que algunos dicen que «dogma es aprobar algo en términos más o menos generales», pues el escéptico asiente a las sensaciones que se imponen a su imaginación; por ejemplo, al sentir frío o claro, no diría «creo que no siento calor» $\mathrm{o}$ «no siento frío». Sino que decimos que no dogmatiza en el sentido en que otros dicen que «dogma es la aceptación en ciertas cuestiones, después de analizadas científicamente, de cosas no manifiestas»; el pirrónico en efecto no asiente a ninguna de las cosas no manifiestas (Sexto, Libro I, cap. VII, 2002: 13).

\section{Si la definición de Hankinson fuera compatible con la actitud escéptica:}

1) Sexto estaría restringido a estados mentales donde «asintiera»o «aceptara»; sin embargo, nunca «creería».

2) Sexto no podría explicar satisfactoriamente el pasaje $\alpha$ desde que sugiere que el escéptico «cree» en un sentido.

3) Sexto estaría comprometido con una definición particular de «creer»y de «aceptar».

Si la definición de Cohen fuera compatible con la actitud escéptica: 
1) Sexto estaría restringido a estados mentales de «aceptación», por lo que no tendría creencia alguna.

2) Sexto estaría comprometido con definiciones establecidas de: «aceptación»y de «creencia».

Si los polifacéticos sentidos de «creencia» de la folk psychology fueran compatibles con la actitud del escéptico:

1) Se le podrían adjudicar creencias al escéptico con base en sus distintos usos, restringiéndolo al uso que refiera a un compromiso fuerte con la verdad o falsedad indubitable de $p$.

2) Se podría explicar coherentemente el pasaje $\alpha$ desde que refiere a usos de dogmata y no a definiciones establecidas.

Suponemos que el escéptico suspendería su juicio sobre definiciones que sugieren un dogma en el sentido de tener un compromiso desmesurado sobre la definición $p$. Para suspender el juicio posiblemente argumentaría lo siguiente:

1) Algunos, como Hankinson y Cohen, defienden que la «creencia» es siempre un juicio sobre aquello que es verdadero; o de manera formal: «creer» parece $F$ para Hankinson y Cohen.

2) Otros, como Frede, defienden que hay dos clases de «creencia»: una en sentido epistemológico, y la otra en sentido no-epistemológico; donde no siempre «creer» implica un juicio sobre aquello que es verdadero; o de manera formal: «creer» parece $-F$ para Frede.

3) Ambas son igual de plausibles.

4) Carecemos de un criterio para decidir entre 1) y 2) (por el argumento [B (1-6)] expuesto al inicio).

5) Suspendemos juicio sobre aquello que es de hecho «creer»; es decir, suspendemos el juicio sobre alguna definición de «creer». 
Del supuesto de que no tenemos una definición unívoca sobre «creencia», podemos decir lo siguiente:

a) La definición de Frede no es compatible desde que se compromete con un concepto sobre aquello que es epistemológico y noepistemológico.

b) La definición de Hankinson no es compatible desde que se compromete con los conceptos de estados mentales como «asentir», «aceptar»y «creer».

c) La definición de Cohen tampoco es compatible por las mismas razones que Hankinson no lo es.

d) La folk psychology al i) no tener una definición que sugiera un dogma en el sentido de tener un compromiso desmesurado sobre $p, \mathrm{y}$ al ii) ser coherente con el pasaje $\alpha$, es la opción más plausible que puede describir la actitud escéptica.

Optar por d), o bien por la folk psychology, implica resguardar el carácter neutral del escepticismo desde que no hay definiciones; y si las hubiera, dependerían del uso (o de la situación particular, relativa, contextual, esto es: las representaciones mentales de las apariencias) que se le dé al término y no de una definición rígida.

\section{Conclusiones}

Es posible atribuirle creencias al escéptico pirrónico si y sólo si no dependemos de una definición acabada de «creencia» que justifique la atribución; por lo que acudir al uso, y no a una definición del término, parece la opción más compatible con lo que practica el escéptico pirrónico, particularmente Sexto Empírico. 


\section{BIBLIOGRAFÍA}

Cohen, L. J. (1989). Belief and acceptance. En Mind. 98-391; 367-89.

Cohen, L.J. (1992). An Essay on Belief and Acceptance. New York: Oxford University Press.

Frede, M. (1987). Essays in Ancient Philosophy. Minneapolis: University of Minnesota Press.

Hankinson, R. J. (1995). The Sceptics: Arguments of the Philosophers. London: Routledge.

Horgan, T. \& Woodward, J. (1985). Folk psychology is here to stay. En The Philosophical Review. 94-2; 197-226.

Hurtado, G. (2009). Dudas y sospechas. En Por qué no soy falibilista y otros ensayos filosóficos. México: Los libros de Homero.

Lina, E. \& Hájek, A. (2007). «What are degrees of belief?». En Studia Logica. 86-2; 183-213.

Oakey, I.T. (1988). Scepticism ans the diversity of epistemic justification. En The Philosophical Quarterly. 38-152; 26379 .

Ravenscroft, I. (2010). Folk Psychology as a Theory. En The Stanford Encyclopedia of Philosophy. Edward N. Zalta (Ed.). En http: / / plato.stanford.edu/archives / fall-2010/ entries / folkpsych-theory / Consultado el 2 de febrero de 2012.

Schwitzgebel, E. (2010). Belief. En The Stanford Encyclopedia of Philosophy. Edward N. Zalta (Ed.). En http:/ / plato. stanford.edu/archives/win2010/entries/belief. Consultado el 2 de febrero de 2012.

Sexto Empírico. (2002). Esbozos pirrónicos. A. Gallego, T. Muñoz (trads). Madrid: Gredos. 


\section{RESUMEN}

Parto del siguiente supuesto: el escéptico pirrónico tiene como objetivo la ataraxia que se consigue a través de la suspensión del juicio - es decir, a través de la suspensión de las creencias sobre el mundo. Analizo distintas definiciones de creencia y juzgo cuál de ellas es la más compatible con lo expuesto por Sexto Empírico; todo esto para responder si el escéptico suspende el juicio de todas sus creencias o sólo de algunas. Finalmente, defenderé que Sexto no se comprometería con una definición restringida de «creer»; por lo que, para atribuirle creencias, tendríamos que optar por un sentido amplio de creencia que dependa de los múltiples usos del término y no de una definición rígida.

Palabras clave: ataraxia, creer, aceptar, folk psychology.

\section{ABSTRACT}

I begin with the following assumption: the pyrrhonian skeptic's aim is ataraxia, which can be achieved by suspending judgment — that is, by suspending all beliefs about the world. I analyze different definitions of belief and I judge which one is the most compatible with Sextus Empiricus's view; all this to asses if the skeptic can suspend the judgment of all its beliefs or just a few of them. Finally, I'll argue that Sextus wouldn't commit to a narrow definition of «belief»; if we want to ascribe him beliefs, we should choose a wider sense of belief that depends on the multiple uses of the term and not just a narrow definition.

Keywords: ataraxia, belief, acceptance, folk psychology. 
